\title{
TIME-DEPENDENT SCHRÖDINGER EQUATION WITH ONE KNOWN SOLUTION
}

\author{
T. V. Fityo, V. M. Tkachuk \\ Ivan Franko National University of Lviv, Department for Theoretical Physics, \\ 12 Drahomanov St., Lviv UA-79005, Ukraine \\ e-mail: fityo@ktf.franko.lviv.ua \\ e-mail: tkachuk@ktf.franko.lviv.ua
}

(Received May 16, 2005; received in final form January 20, 2006)

\begin{abstract}
We present a method to construct a time-dependent Schrödinger equation with one known solution. Several explicit examples are presented. The quantum Kapitsa effect is discussed.

Key words: (quasi-)exactly solvable potential, time-dependent Schrödinger equation.
\end{abstract}

PACS number(s): 02.30.Zz, 03.65.Ge

\section{INTRODUCTION}

In this paper we consider 1D time-dependent Schrödinger equation:

$$
i \frac{\partial}{\partial t} \psi=H \psi=-\frac{\partial^{2}}{\partial x^{2}} \psi+V(x, t) \psi
$$

We assume that function $\psi(x, t)$ belongs to the space where operator $H$ is Hermitian and that $\psi(x, t)$ can be normalized to unity.

It is impossible to solve equation (1) for the general form of potential $V(x, t)$. But there is a possibility to construct such a potential that for the corresponding Schrödinger equation all solutions or only some of them can be found.

We would like to outline three approaches to this problem. The first one, developed by Lewis and Riesenfeld [1], can be applied to special classes of quantum systems (e. g., harmonic oscillator, harmonic plus inverse harmonic potential [2], linear potential [3], etc.). Instead of solving the corresponding Schrödinger equation directly the so-called invariant is found and on the basis of its eigenfunctions a general solution of Schrödinger equation (1) is constructed.

The second approach is based on Darboux transformation $[4,5]$. In this case, to time-dependent or timeindependent Schrödinger equation with known solutions Darboux transformation is applied to obtain a new timedependent Schrödinger equation with known solutions.

The third approach is based on point transformation $[6,7]$. In this case one chooses new coordinates in such a way that a new equation of Schrödinger type can be obtained from the old one that is exactly solvable. There are simple connections between potentials of the equations. These connections give a possibility to find solutions of the new Schrödinger equation knowing solutions of the old equation.

In this article we will use the inverse method [8] to construct an equation of type (1) with one known solution. A realization of this method in our article is quite simple: we choose such a normalizable solution that the corresponding potential is real.

\section{CONSTRUCTING THE POTENTIAL}

Making the following ansatz $\psi(x, t)=e^{-f(x, t)-i g(x, t)}$ into equation (1) we obtain

$$
V=g_{t}+f_{x}^{2}-g_{x}^{2}-f_{x x}+i\left(2 f_{x} g_{x}-f_{t}-g_{x x}\right),
$$

where $f$ and $g$ are real functions, function $f$ provides square integrability of wave-function $\psi$; subscript indices $t$ and $x$ denote partial derivatives with regards to $t$ and $x$ respectively.

Requiring reality of the potential we obtain the following condition

$$
f_{t}+g_{x x}=2 f_{x} g_{x}
$$

In this case the potential reads

$$
V(x, t)=g_{t}+f_{x}^{2}-g_{x}^{2}-f_{x x}
$$

For fixed $f$ we can easily solve equation (3) with respect to $g$. The solution reads

$$
\begin{aligned}
& g(x, t)=c_{1}(t) \int_{-\infty}^{x} e^{2 f(y, t)} d y \\
& +\frac{1}{2} \int_{-\infty}^{x} e^{2 f(y, t)} \frac{\partial}{\partial t} \int_{-\infty}^{y} e^{-2 f(z, t)} d z d y+c(t),
\end{aligned}
$$

where $c_{1}(t)$ and $c(t)$ are arbitrary real functions of $t$ (they are constants of integration over $x$ ).

Let us analyze two special cases when either the potential or the particle's density does not depend on time.

Case 1.

The potential is a function of only $x: V(x, t)=V(x)$. It is well known that for such time-independent potentials a general solution of equation (1) can be expressed in the following form [9]: 


$$
\psi(x, t)=\sum_{\alpha} C_{\alpha} e^{-i E_{\alpha} t} \phi_{\alpha}(x)
$$

where $\phi_{\alpha}(x)$ and $E_{\alpha}$ are eigenfunctions and appropriate eigenvalues of operator $H$. So, in this case the problem of constructing time-dependent Schrödinger equation is reduced to the problem of constructing timeindependent Schrödinger equation, which is well investigated $[8,10-12]$.

Case 2.

$f(x, t)=f(x)$. This corresponds to the case that the particle's density $\rho(x, t)=e^{-2 f}$ does not depend on time. Solution (5) can be simplified as

$$
g(x, t)=c_{1}(t) \int_{-\infty}^{x} e^{2 f(y)} d y+c(t)
$$

Now we should determine if our choice of $f$ and $g$ leads to the wave-function $\psi$ belonging to a space where the Hamiltonian is an Hermitian operator. Operator $H$ contains $p^{2}$ term, so for any satisfactory $\psi$ there exists averaged value of kinetic energy $\left\langle p^{2}\right\rangle$ and $\left\langle p^{2}\right\rangle \geq\langle p\rangle^{2}$. Therefore, $\langle p\rangle$ must exist too. To consider whether this integral exists we estimate $-i \psi^{*}(x, t) \frac{\partial}{\partial x} \psi(x, t)$ at infinity.

$$
\begin{aligned}
& -i \psi^{*}(x, t) \frac{\partial}{\partial x} \psi(x, t)=i f_{x}(x) e^{-2 f} \\
& -c_{1}(t) \underset{x \rightarrow \pm \infty}{\longrightarrow}-c_{1}(t) .
\end{aligned}
$$

Here we assume that $f(x)$ does not oscillate very fast and it tends to infinity as $x$ tends to infinities to satisfy square integrability of the wave function. So we can neglect the first term. If $c_{1}(t) \neq 0$, then $\langle p\rangle$ diverges and $\psi(x, t)$ does not belong to the space where $H$ is an Hermitian operator.

We also would like to note that the obtained result is equivalent to the requirement that the wave function $\psi$ is square integrable and it does not oscillate very rapidly (i. e., $f$ has a smooth character and the absolute value of $g$ does not become too large) with a change of the spatial coordinate.

So, the sole satisfactory solution (7) of equation (3) is the case of $c_{1}(t)=0$ and $g(x, t)=c(t)$ and potential has the following form

$$
V(x, t)=c_{t}(t)+f_{x}^{2}(x)-f_{x x}(x),
$$

and solution $\psi(x, t)$ has the form

$$
\psi(x, t)=e^{-f(x)-i c(t)} .
$$

The time-dependence of solution (10) and the corresponding potential (9) can be eliminated via an obvious unitary transformation $U=e^{i c(t)}$ so the problem is also equivalent to the time-independent one.
A general case.

Using equation (5) we estimate $-i \psi^{*}(x, t) \frac{\partial}{\partial x} \psi(x, t)$ at infinities once more.

$$
\begin{aligned}
& -i \psi^{*}(x, t) \frac{\partial}{\partial x} \psi(x, t)=i f_{x}(x) e^{-2 f(x, t)} \\
& -c_{1}(t)-\frac{1}{2} \frac{\partial}{\partial t} \int_{-\infty}^{x} e^{-2 f(y, t)} d y
\end{aligned}
$$

At $x \rightarrow \pm \infty$ the first term vanishes. So at $x \rightarrow-\infty$ (11) tends to $-c_{1}(t)$ and thus we must set $c_{1}(t)=0$. At $x \rightarrow \infty$ (11) tends to $-\frac{1}{2} \frac{\partial}{\partial t} \int_{-\infty}^{x} e^{-2 f(y, t)} d y$ which must be zero too. Thereby, we have to choose $f$ in such a way that $\int_{-\infty}^{\infty} e^{-2 f(y, t)} d y$ is a time-independent constant (unity as a constant is a natural choice). In order to satisfy time-independence of the mentioned integral we rewrite $f$ as follow

$$
\begin{gathered}
f(x, t)=\tilde{f}(x, t)+\frac{1}{2} \ln F(t), \\
F(t)=\int_{-\infty}^{\infty} e^{-2 \tilde{f}(x, t)} d x
\end{gathered}
$$

where $\tilde{f}(x, t)$ is an arbitrary function for which $F(t)$ is convergent.

For such a choice of function $f(x, t)$ the wave function $\psi(x, t)$ is normalized to unity. Having $f(x, t)$ we can calculate $g(x, t)$ using equation (5) and putting $c_{1}(t) \equiv 0$ as well as potential $V(x, t)$ using equation (4).

\section{EXAMPLES}

We can easily find $g(x, t)$ if $\tilde{f}$ is written as

$$
\tilde{f}(x, t)=\tilde{f}(a(t) x+b(t))
$$

To ensure normalizability of function $\psi$ we require that $a(t)$ should not change the sign. Then equation (5) gives

$$
g(x, t)=\frac{a^{\prime}(t)}{4 a(t)} x^{2}+\frac{b^{\prime}(t)}{2 a(t)} x+c(t) .
$$

Note that in case (12) the corresponding operator $H$ can be transformed using point transformation into timeindependent Schrödinger operator [13]: if we introduce a new coordinate and a new time

$$
\bar{x}=a(t) x+b(t), \quad \bar{t}=\int^{t} a(s)^{2} d s
$$

then the new solution

$$
\bar{\psi}(\bar{x}, \bar{t})=e^{\frac{i}{4}\left(\frac{a^{\prime}}{a} x^{2}+2 \frac{b^{\prime}}{a} x+4 c\right)} \psi(x, t)
$$


and the new potential

$$
\begin{aligned}
& \bar{V}(\bar{x})=\frac{1}{a^{2}} V(x, t)+\frac{x^{2}}{4 a^{2}}\left(2 \frac{a^{\prime 2}}{a}-a^{\prime \prime}\right) \\
& -x\left(\frac{b^{\prime \prime}}{2 a^{3}}-b^{\prime} a^{\prime}\right)+\frac{b^{\prime 2}}{4 a^{4}}+\frac{c^{\prime}}{a^{2}}
\end{aligned}
$$

correspond to the time-independent Schrödinger equation (potential (15) does not depend on the new time $\bar{t})$.

Examples 1 and 2 illustrate this case. The third example technically is more complicated.

Example 1.

Let us choose $\tilde{f}$ in the form of

$$
\tilde{f}=\left(1+t^{2}\right) x^{2}
$$

then

$$
\begin{gathered}
f=\tilde{f}+\frac{1}{4} \ln \left(\frac{\pi}{2\left(1+t^{2}\right)}\right), \\
g=\frac{1}{4} \frac{x^{2} t}{1+t^{2}} \\
V(x, t)=\left[\frac{1-3 t^{2}}{4\left(1+t^{2}\right)^{2}}+4\left(1+t^{2}\right)^{2}\right] x^{2}-2\left(1+t^{2}\right) .
\end{gathered}
$$

The expression in brackets is always positive. The corresponding function reads

$$
\psi(x, t)=\left(\frac{2\left(1+t^{2}\right)}{\pi}\right)^{\frac{1}{4}} e^{-\left(1+t^{2}\right) x^{2}-\frac{i}{4} \frac{x^{2} t}{1+t^{2}}}
$$

The larger $|t|$ becomes the more particle density increases at the origin.

The average energy can be calculated as

$$
\langle E\rangle=\left\langle i \frac{\partial}{\partial t}\right\rangle=\frac{1}{16} \frac{1-t^{2}}{\left(1+t^{2}\right)^{3}} .
$$

\section{Example 2.}

Let us choose $\tilde{f}$ in the form of

$$
\tilde{f}=x(1+a \sin \omega t)+e^{-2 x(1+a \sin \omega t)},
$$

where $|a|<1$. Then

$$
\begin{aligned}
& f=\tilde{f}-\frac{1}{2} \ln 4(1+a \sin \omega t), \\
& g=\frac{1}{4} \frac{x^{2} a \omega \cos \omega t}{1+a \sin \omega t},
\end{aligned}
$$

$$
\begin{aligned}
V(x, t)= & -\left[\frac{a \omega^{2} \sin \omega t}{1+a \sin \omega t}+2 \frac{a^{2} \omega^{2} \cos ^{2} \omega t}{(1+a \sin \omega t)^{2}}\right] \frac{x^{2}}{4} \\
& -4(1+a \sin \omega t)^{2} e^{-2 x(1+a \sin \omega t)}+(1+a \sin \omega t)^{2}\left(1-2 e^{-2 x(1+a \sin \omega t)}\right)^{2}
\end{aligned}
$$

Function (19) is a $\frac{2 \pi}{\omega}$ periodic in time and it is a square integrable function for any $\omega$.

The average energy for this system reads

$$
\begin{aligned}
\langle E\rangle & =\left\langle i \frac{\partial}{\partial t}\right\rangle=-\frac{a \omega^{2}}{(1+a \sin \omega t)^{4}}(a+\sin \omega t) \\
& \times \int_{-\infty}^{\infty} x^{2} e^{-2 x-2 e^{-2 x}} d x .
\end{aligned}
$$

The value of the last integral is $\pi^{2} / 96+(\gamma+\ln 2)^{2} / 16 \approx$ 0.20367 , where $\gamma$ is Euler's constant.
In the limit $a \rightarrow 0, \omega=\frac{2}{a}$ we can find averaged value of potential (18).

$$
\begin{aligned}
& \langle V(x, t)\rangle_{t}=\frac{\omega}{2 \pi} \int_{0}^{\frac{2 \pi}{\omega}} V(x, t) d t= \\
& -\frac{x^{2}}{2}-4 e^{-2 x}+\left(1-2 e^{-2 x}\right)^{2} .
\end{aligned}
$$

This oscillating potential can describe a particle's behaviour in one-dimensional Paul-like trap [14]. 


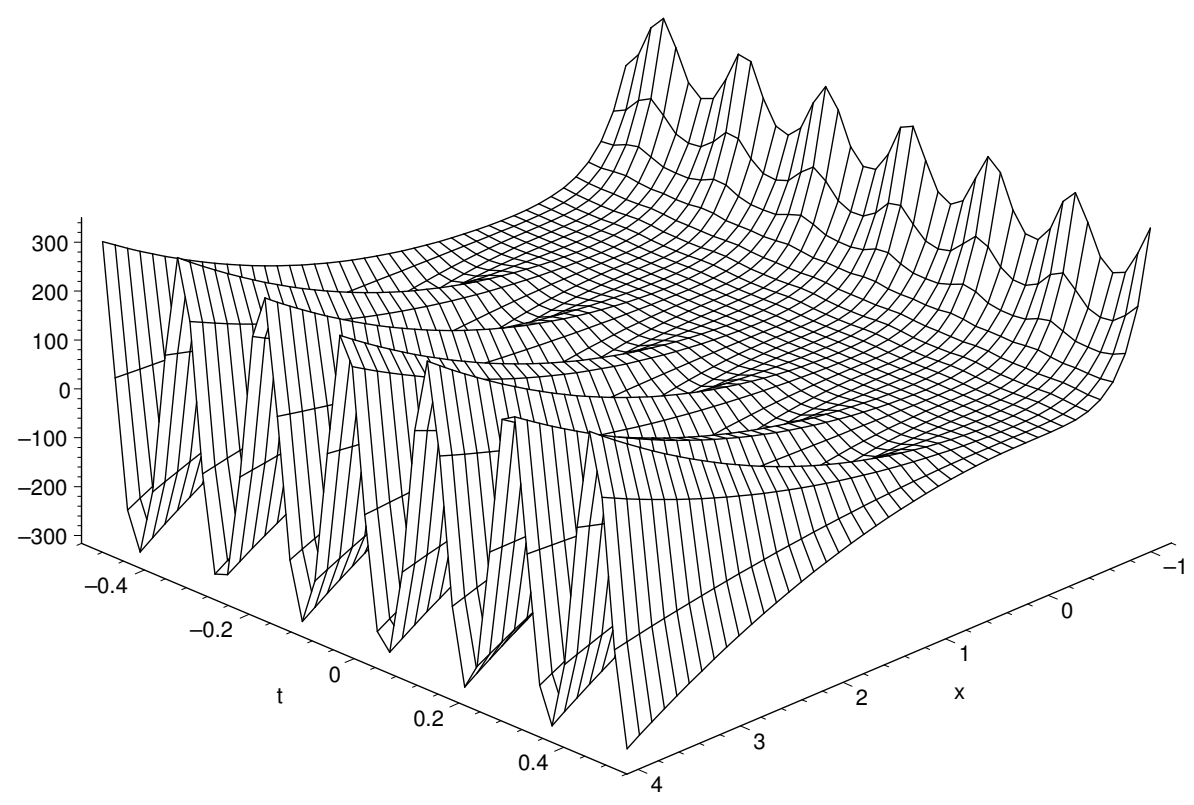

Fig. 1. Potential (18) for $a=\frac{1}{20}, \omega=40$.

We can observe an interesting effect: there are no bound states for the averaged potential (20), but little rapid oscillations of the particle's coordinate entrap this particle. As we mentioned above similar systems are known as Paul traps. The mathematics describing Paul traps is quite complicated, but there exists a simpler approximate approach known as the quantum Kapitsa effect [15]. Let us compare our exact result with the approximated one given in [15].

According to that paper, if potential $V$ contains rapidly oscillating terms $U$, then spatial properties of a particle are governed by the time-independent Schrödinger equation with the effective potential

$$
V_{\mathrm{eff}}(x)=\langle V\rangle_{t}+\frac{f_{1}^{2}(x)+f_{2}^{2}(x)}{2 \Omega^{2}},
$$

where $\Omega$ is frequency of oscillations and $\frac{\partial}{\partial x} U=$ $f_{1} \cos \Omega t+f_{2} \sin \Omega t$. For potential (18) $\frac{\partial}{\partial x} U=x \omega \sin \omega t$. So,

$$
V_{e f f}(x)=-4 e^{-2 x}+\left(1-2 e^{-2 x}\right)^{2}
$$

This potential is the so-called Morse potential and it is an exactly solvable one $[16,17]$. In contrast to the averaged potential, effective potential (22) has one bound state

$$
\psi(x, t)=2 e^{-x-e^{-2 x}}
$$

with eigenvalue equaling 0 . It is interesting to note, that point transformation (13) leads to the same potential (22) and the same wave function (23). Thus, the quan- tum Kapitsa approximation [15] is quite good for potential (18).

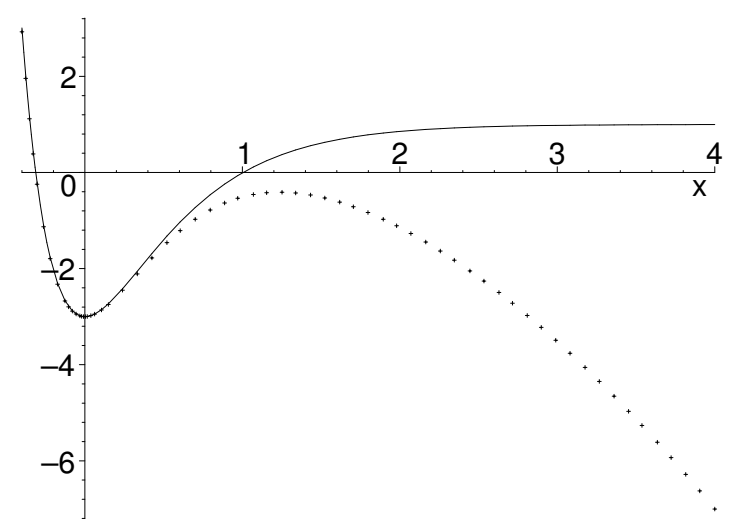

Fig. 2. Averaged potential (20) - doted line, effective potential $(22)$ - solid line.

\section{Example 3.}

In previous examples $\tilde{f}$ was of the form of (12). Here we choose $\tilde{f}$ to be in a more complicated form.

$$
\tilde{f}=\frac{1+t^{2}}{2} \sinh ^{2} x-\frac{1}{2} \ln \cosh x,
$$

then

$$
\begin{gathered}
f=\frac{1}{4} \ln \frac{\pi}{1+t^{2}}+\tilde{f} \\
g=\frac{t \ln \cosh x}{2\left(1+t^{2}\right)}
\end{gathered}
$$


As a result we obtain the following expressions for the potential energy and the corresponding wave function:

$$
\begin{aligned}
V(x, t) & =\frac{\left(1-t^{2}\right) \ln \cosh x}{2\left(1+t^{2}\right)^{2}}-\frac{t^{2} \tanh ^{2} x}{4\left(1+t^{2}\right)^{2}} \\
& +\left(\frac{\left(1+t^{2}\right) \sinh 2 x-\tanh x}{2}\right)^{2}-\left(1+t^{2}\right) \cosh 2 x+\frac{1}{2 \cosh ^{2} x} \\
\psi(x, t) & =\sqrt[4]{\frac{1+t^{2}}{\pi} \cosh ^{2} x} \exp \left\{-\frac{1+t^{2}}{2} \sinh ^{2} x-\frac{i t}{2\left(1+t^{2}\right)} \ln \cosh x\right\}
\end{aligned}
$$

When $|t|$ increases the form of the potential energy becomes more and more narrow and particle density of probability increases at the origin.

In contrast to the previous examples this example cannot be reduced to the time-independent form using pointtransformation (13).

\section{CONCLUDING REMARKS}

In this paper we have developed a simple approach for constructing time-dependent Schrödinger equation with one known solution. The real function $f(x, t)$ plays a role of the generation function. By choosing different appro- priate functions $f$ we can generate potentials with one known solution. This is demonstrated by examples.

At the end of this paper we would also like to discuss the number of known solutions of the Schrödinger equation constructed in such a way. For the time-independent one dimensional case each consequent solution is real and requires one additional condition (every solution must lead to the same potential). Thus, for this case it is quite easy to build time-independent Schrödinger equation with two known levels $[10,11]$ or even with three levels [12]. Conversely, for our case each solution is complex one requiring an additional condition because of the reality of the potential. That is why we managed to construct Schrödinger equation with only one known solution.
[1] H. R. Lewis, Jr., W. B. Riesenfeld, J. Math. Phys. 10, 1458 (1969).

[2] R. S. Kaushal, D. Parashar, Phys. Rev. A 55, 2610 (1997).

[3] J. Q. Shen, preprint quant-ph/0310179 (2003).

[4] V. G. Bagrov, B. F. Samsonov, Phys. Lett. A 210, 60 (1996).

[5] F. Cannata et al., J. Phys. A: Math. Gen. 32, 3583 (1999).

[6] G. Bluman, V. Shtelen, J. Phys. A: Math. Gen. 29, 4473 (1996).

[7] A. Mostafazadeh, J. Phys. A: Math. Gen. 31, 6495 (1998).

[8] A. G. Ushveridze, Quasi-Exactly Solvable Models in Quantum Mechanics (Institute of Physics, Bristol, 1994).

[9] L. D. Landau, E. M. Lifshitz, Quantum mechanics: non- relativistic theory (Pergamon Press, Oxford, New York, 1977).

[10] V. M. Tkachuk, Phys. Lett. A 245, 177 (1998).

[11] S. N. Dolya, O. B. Zaslavskii, J. Phys. A: Math. Gen. 34, 1981 (2001).

[12] T. V. Kuliy, V. M. Tkachuk, J. Phys. A: Math. Gen. 32, 2157 (1999).

[13] F. Finkel et al., J. Math. Phys. 40, 3268 (1999).

[14] W. Paul, Rev. Mod. Phys. 62, 531 (1990).

[15] R. J. Cook, D. G. Shankland, A. L. Wells, Phys. Rev. A 31, 564 (1985).

[16] F. Cooper, A. Khare, U. Sukhatme, Phys. Rep. 251, 267 (1995).

[17] M. M. Morse, H. Feshbach, Methods of Theoretical Physics (McGraw Hill, New York, 1953), Vol. II.

\section{ЧАСОВО-ЗАЛЕЖНЕ РІВНЯННЯ ШРЕДИНГЕРА $З$ ОДНИМ ВІДОМИМ РІВНЕМ}

Т. В. Фітьо, В. М. Ткачук

Лъвівсъкий начіональний університет імені Івана Франка, кафедра теоретичної фізики, вул. Драгоманова, 12, Лъвів, 79005, Україна

Запропоновано метод побудови часово-залежного рівняння Шредингера з одним відомим розв'язком. Наведено декілька явних прикладів. Проаналізовано квантовий ефект Капіци. 